\title{
EELS Estimation of Ice Accumulation Rates in a Cryo-TEM Equipped with a Multi-Sample Loading Device.
}

\author{
Agustin J. Avila-Sakar. ${ }^{1}$ \\ ${ }^{1}$ Department of Biological Sciences, Purdue University, West Lafayette, Indiana.
}

The contrast in low dose images of biological macromolecules embedded in vitreous ice depends in part on the thickness of the ice layer in which they are contained. In spite of a complicated dependence on a variety of physicochemical parameters that affect the flash-freezing process used to prepare samples for cryo-TEM, the resulting ice thickness is nowadays easier to control by use of robotic freezing apparatuses. Nevertheless, once in the microscope, the low water vapor pressure in the evacuated spaces of the instrument sustain the growth of a layer of amorphous ice on the samples. The increased thickness reduces the contrast of ice embedded macromolecules, ultimately affecting the resolution and setting a limit to the time over which an experiment can be extended [1]. On microscopes equipped with multi-sample loading devices, a process of accumulation also takes place in the chamber where samples sit temporarily while their turn comes to be transferred into the column.

EELS is a technique commonly used to measure the relative and absolute thickness of thin layers of materials in the electron microscope. The relevant information resides in 1) the relationship between the zero-loss peak and the region of the spectrum representing the contribution of all scattered electrons that have lost energy upon interaction with the specimen; 2) the elemental composition of the specimen; 3) the differential ionization cross-sections for the chemical elements present; and 4) experimental parameters, such as beam convergence and collection angle. In this study, the relevant elements were carbon and oxygen. Spectra acquisition and analysis was done using Digital Micrograph (Gatan, Inc.).

A Quantifoil EM grid with a thin film of continuous carbon spanning the holes of the perforated pattern of the grid, but without a specimen, was loaded dry into the autoloader of a Krios Electron Microscope (FEI Company) and stored there for 44 hours, then transferred into the column. EELS spectra were recorded over a selected area of the thin carbon film using a Tridiem energy filter equipped with a $2 \mathrm{~K}$ Ultrascan CCD camera (Gatan, Inc.). The area was first cleaned of any ice so far accumulated by illuminating it for a couple of minutes with a relatively intense beam. Immediately thereafter a control spectrum of the ice-free area was recorded. New spectra were recorded at various time intervals over a span of 85 hours. Finally, the area was cleaned again of the accumulated ice by letting the grid warm up to room temperature and a second control spectrum was then recorded.

Signal from the oxygen K core-loss edge was observed in spectra from areas that had accumulated ice and could be eliminated by exposure to a high-intensity beam, leaving only the carbon signal from the thin continuous film (Fig 1). The oxygen signal reappeared and grew with time as ice condensed again on the cold carbon surface. The relationship between the areas under the oxygen and carbon edges yielded a measure of the relative abundance of those elements. The proportion of oxygen to carbon increased linearly with time, and so did the relative thickness $(t / \lambda)$ of the entire layer, as derived from the low-loss region of the spectra. Since the carbon film is unaffected by the experiment, the increase in oxygen content must correspond to the increase in the thickness of the ice layer. The absolute thickness of the thin supporting carbon film was estimated from $t / \lambda$ measurements when the ice had been removed either by an intense beam or by warming up the sample to room temperature. The difference between the 
two measurements was $0.09 \mathrm{~nm}$, which is interpreted as statistical error, and the average of the two measurements was $9.58 \mathrm{~nm}$. The thickness of the accumulating ice layer was estimated from the elemental ratio of carbon to oxygen, and from the molar volume ratio of carbon to amorphous ice. For the latter ratio, the density of carbon was taken as $2.0 \mathrm{~g} / \mathrm{cm}^{3}$ [2], and that of vapor-deposited amorphous ice as $0.82 \mathrm{~g} / \mathrm{cm}^{3}$ [3]. The linear fit to the estimated thickness gave an accumulation rate of $0.77 \mathrm{~nm} / \mathrm{h}$ in the column (Fig 2). The initial ice content of the observed area (before it was removed with an intense beam) was estimated in the same way. Since loading times in autoloader and column were known, it was also possible to infer the rate of ice accumulation in the autoloader, which turned out to be 0.41 $\mathrm{nm} / \mathrm{h}$ (Fig 2).

Knowledge of the ice accumulation rates is useful for the planning of high-throughput cryo-TEM experiments, and, more generally, for evaluating the adequacy of an instrument for such lengthy tasks. On microscopes equipped with energy filters, the data needed to estimate such rates can be extracted from only a few spectra, making it a relatively simple process involving few steps.

\section{References:}

[1] Cheng et al, J. Struct. Biol. 154 (2006), p. 303.

[2] Logothetidis S. and Stergioudis G., Appl. Phys. Lett. 71 (1997), p. 2463

[3] Brown D.E. et al, J. Phys. Chem. 100 (1996), p. 4988.

[4] The Krios cryo-TEM was acquired with a NIH RR grant for major research instrumentation to Michael Rossmann, plus matching funds from Purdue University.

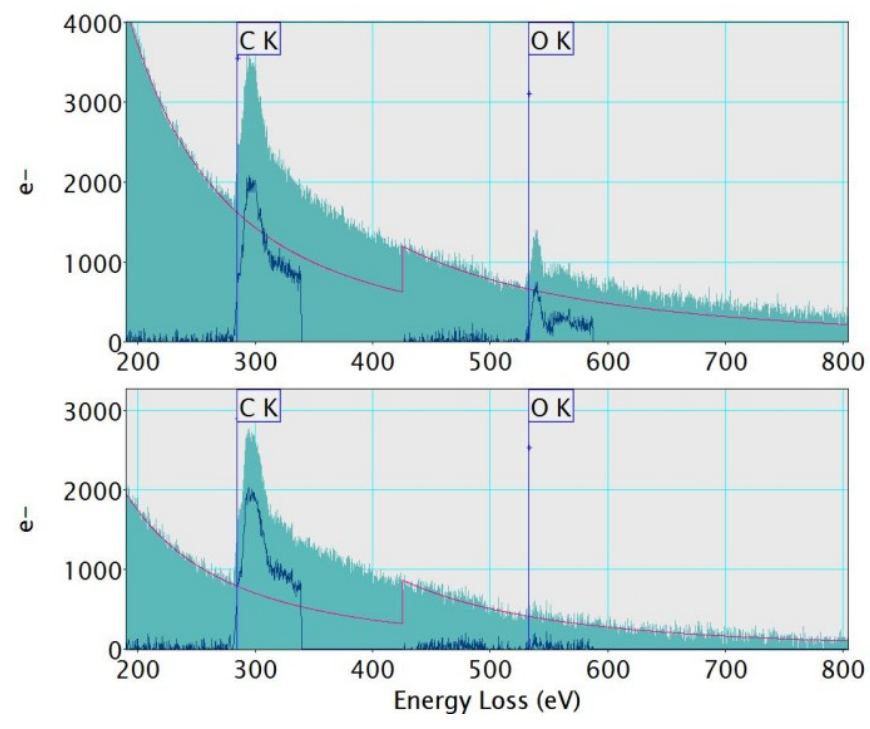

Figure 1. EELS spectra including the carbon and oxygen core-loss regions. Upper panel shows oxygen signal from accumulated ice. Oxygen signal is absent in lower panel, after ice has been removed by exposure to intense beam.

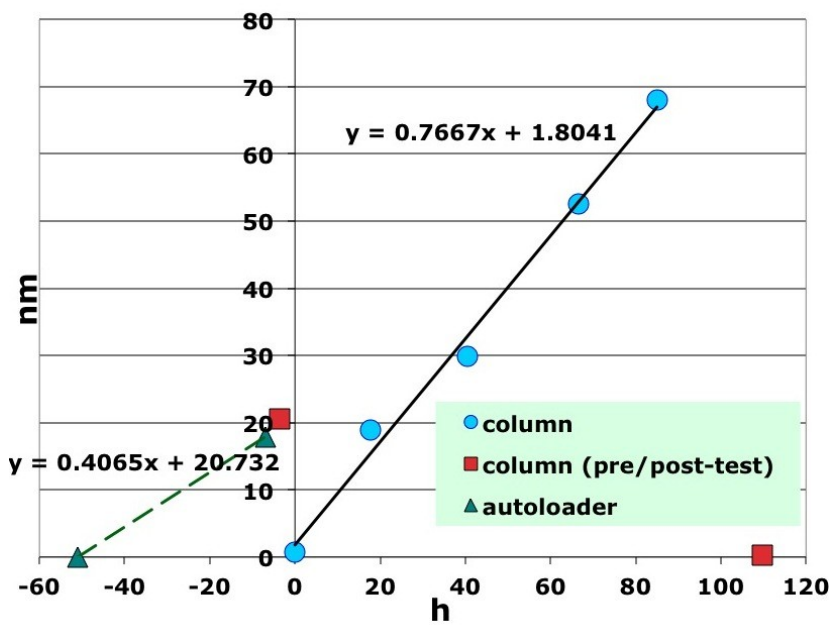

Figure 2. Time course of ice accumulation: Thickness (nm) vs time (h). Red datum near center was recorded immediately after sample insertion into the column. First blue circle and last red square are data from plain carbon film, where the ice had been removed by intense beam or warm up to room temperature, respectively. 